\title{
TECCIENCLA
}

\section{Evaluation of the Performance of Techniques to Transmit IPv6 Data through IPv4 Networks}

\author{
Evaluación del desempeño de las técnicas para la transmisión de datos IPv6 a través de redes \\ IPv4
}

\author{
Nancy Yaneth Gelvez García ${ }^{1}$, Juan Manuel Sánchez Céspedes ${ }^{2}$, Jhon Francined Herrera Cubides ${ }^{3}$ \\ ${ }^{1}$ Universidad Distrital Francisco José de Caldas, Bogotá, Colombia, nygelvezg@ udistrital.edu.co \\ ${ }^{2}$ Universidad Distrital Francisco José de Caldas, Bogotá, Colombia, jmsanchezc@ udistrital.edu.co \\ ${ }^{3}$ Universidad Distrital Francisco José de Caldas, Bogotá, Colombia, jfherrerac@udistrital.edu.co
}

\begin{abstract}
This article presents the simulation of an IPv4 network connected to two IPv6 isles. Those protocols are not compatible; therefore, transition mechanisms were implemented to fulfill a fundamental role. Meanwhile, this reaches the total deployment of IPv6, such as: Tunneling and Address translation. The first, encapsulates an IPv6 packet inside an IPv4 Header so that it can be moved through the network; the second, translates the address and the protocol of those packets crossing through it. To assess the performance of each technique when the data is sent, the changes with respect to memory consumption and router processing were analyzed.
\end{abstract}

Key words: RFCs, transition mechanisms, Tunneling, address translation

\section{Resumen}

Este artículo presenta la simulación de una red IPv4 conectada a dos islas IPv6. Estos protocolos no son compatibles; por lo tanto, se implementaron mecanismos de transición que cumplen un papel fundamental mientras se logra el despliegue total de IPv6, como: tunneling y traducción de direcciones. El primero encapsula un paquete IPv6 en una cabecera IPv4 para que pueda ser transportado por la red; el segundo traduce las direcciones y el protocolo de aquellos paquetes que pasan a través de él. Para evaluar el desempeño que posee cada técnica al momento de enviar datos, se analizaron los cambios respecto a consumo de memoria y al procesamiento de los enrutadores.

Palabras claves RFC's, mecanismo de transición, tunneling, traducción de direcciones

\section{Introduction}

In 1977, Americans Vinton Cerf and Robert Kahn, recognized as the "internet pioneers" created the IPv4 protocol. They established a range of over 4.3 billion IP addresses for computers to be able to connect amongst themselves in the networks. However, they never imagined that internet would have an exponential growth, which produced a significant increase of the internet during the 1990s; expanding rapidly through society, faster than telephones and faxes.

In a relatively short period of time, the IPv4 protocol will be inefficient to operate the internet network due to the depletion of IP addresses, given that the Internet Assigned Numbers Authority (IANA), on 3 February 2011, assigned the last blocks of IP addresses v4/8 to each department of regional internet registries (RIRs) in the world [1].

This made it necessary to create a new protocol called IPv6 or also called new generation protocol, but if the change was from IPv4 to IPv6, what happened to IPv5? Simply, IANA designated IPv5 as an experimental protocol called "Stream Protocol version 2 (ST-II)", the idea was to recognize an STII packet by observing the IP protocol version number: 4 was a normal packet and 5 was an ST-II packet, for this reason the new version is IPv6 and not IPv5 [2]

The IPv6 protocol is designed to replace the IPv4 protocol; the principal characteristic the new protocol has is the expansion 


\section{TECCIENCIA}

of address space, given that it has $2^{128}$ IP addresses available, while its predecessor has $2^{32}$, that is, the IPv6 quadruples IPv4 in number of addresses [3]. Additionally, IPv6 includes greater efficiency in the delivery of packets, support for communication security, among others.

Bearing in mind that the current infrastructure was designed for IPv4 and not IPv6, mechanisms or methods are used that permit the compatibility of these two protocols so they can use the same infrastructure.

Among these transition mechanisms, there are the Tunneling type and address translation mechanisms. The first transition technique is called Tunneling; this mechanism consists in sending IPv6 frames with an IPv4 header. The tunnel ends are in charge of the header for the packet to use the IPv4 infrastructure. The second transition technique is known as address translations; this mechanism assigns transparent routes in the nodes of the IPv6 isles to communicate with the IPv4 networks and vice versa.

Currently, in Colombia the Renata company is the pioneer in implanting IPv6 and adopted the following address: (2001:13f8::/32). Renata has configured over 70 higher education institutions, which send data through this protocol [4].

\section{Materials and methods}

The following programs are required:

- GNS3 Emulator + Dynamips

- Wireshark

\section{1 general diagram}

The development of this project encompasses transition mechanisms like: Tunneling and Address translation, simulated in GNS3 to evaluate the performance in relation to latency when sending data, memory and CPU consumption when an IPv6/IPv4 node encapsulates or translates a packet.

The development of this work considered the guidelines provided by the RFCs. The RFCs is a group of documents on the internet, these serve to specify, describe and aid in the implementation, standardization, and debate of norms; besides standards, protocols, and technologies related to the internet [5].

\subsection{RFC 4213 transition mechanisms}

The mechanisms used were:

Tunneling: uses tunnels to transport IPv6 packets on IPv4 networks, where the IPv6 packets are encapsulated within an IPv4 header so they can travel the current infrastructure.

Address translation: the protocol translators fulfill the function of translating the IPv6 packets into IPv4 and vice versa, creating transparent routes in the networks of the IPv6 nodes to communicate them to IPv4 nodes [6].

\subsection{RFC 4213 Tunneling}

This transition mechanism provides a way of using the large existing IPv4 infrastructure to carry IPv6 traffic. To apply this mechanism, it can be used in the following manner:

Router to Router: IPv6/IPv4 routers interconnected to an IPv4 infrastructure can receive and send IPv6 packets. In this case, each end of the tunnel configures the destination and origin addresses for IPv6 and IPv4 [7]. The Tunneling techniques are generally classified according to the mechanisms through which the encapsulating node determines the address of the tunnel's final node, in the methods mentioned (router to router and host to host), the final node fulfills the de-encapsulating function of the IPv6 packet so it can be delivered to its destination [8].

The following specifies each of the types of tunnels:

\subsubsection{Manual tunnel}

For manual Tunneling, the address at the end of the tunnel is determined from the encapsulation node information, it should store the address from the tunnel's end point. When an IPv6 packet is sent through the tunnel, the address from the tunnel end is used to encapsulate the IPv4 header

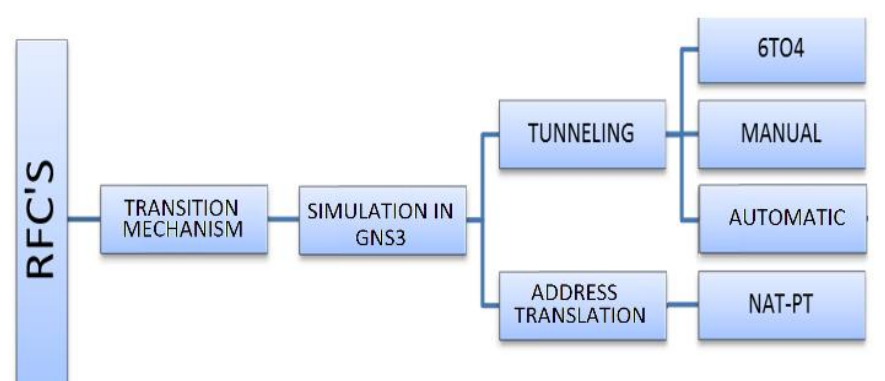

Figure 1. General diagram of the simulation 


\section{TECCIENCIA}

\section{- Topology in GNS3}

Figure 2 shows the topology of a manual tunnel; in this case, Tunneling takes place from router to router (R1 to R4).

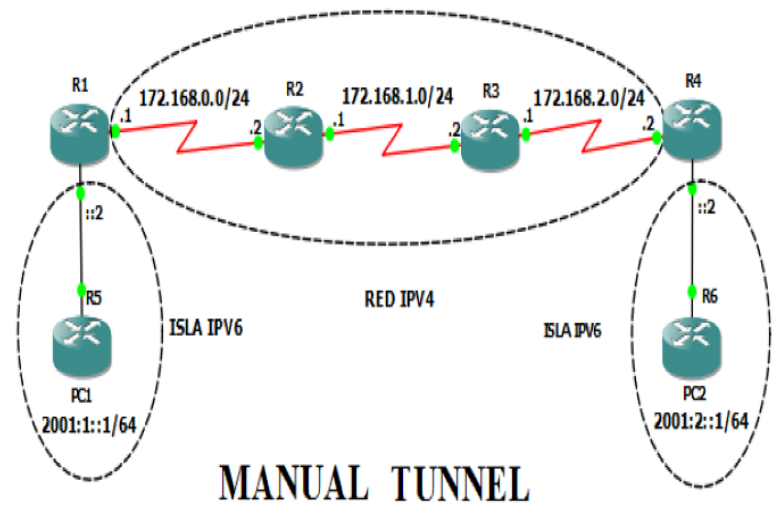

Figure 2. Manual tunnel topology in GNS3

The $\mathrm{R} 5$ and $\mathrm{R} 6$ routers act as $\mathrm{PCs}$, the $R 1$ and $R 4$ routers are IPv6/IPv4 in which the tunnel is configured for the IPv6 packets to reach their destination. The $R 2$ and $R 3$ routers are only IPv4.

\section{- Tunnel configuration}

The main parameters required are the tunnel input and output so IPv6 packets can travel. Figure 3 shows how a tunnel is created (in R1 and R4).

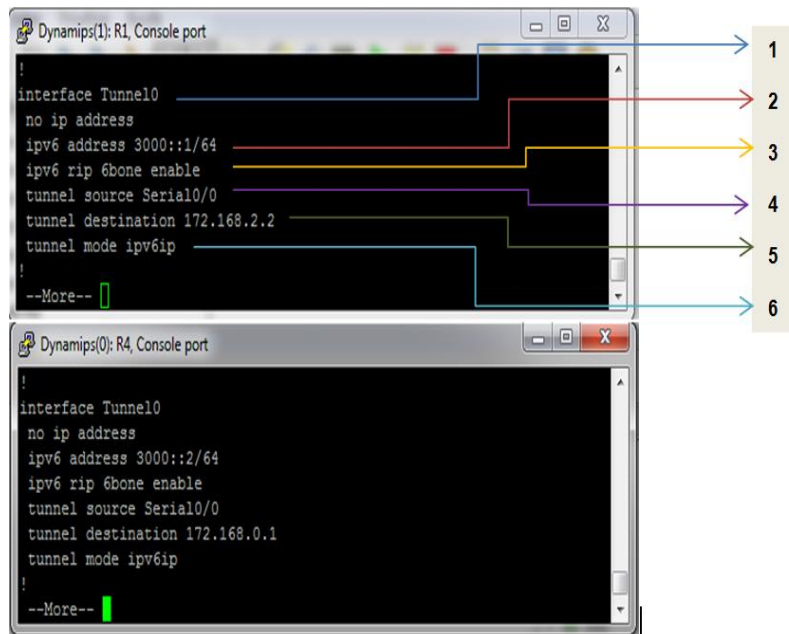

Figure 3. Manual tunnel configuration

The following explains the numerals contained in $\mathrm{R} 1$ (Figure 3).

(1) Interface tunnel0: the tunnel is created; in this case a value of 0 is assigned.
(2) IPv6 Address $3001:: 1 / 64$ : it is the tunnel IPv6 address in $\mathrm{R} 1$.

$>$ (3) IPv6 RIP 6bone enable: enables the RIP protocol RIP in IPv6.

(4) Tunnel source serial0/0: indicates the tunnel source in IPv4; for this case the serial0/0.

(5) Tunnel destination 172.168.2.2: indicates the tunnel's final destination; in this case IPv4 address 172.168.2.2 in which it is the serial0/0 of the $R 4$ router.

(6) IPv6IP Tunnel mode: indicates that the IPv6 tunnel will be created on IPv4.

For the R4, the same logic is managed as in $\mathrm{R} 1$ to create the tunnel and, thus, communicate the two IPv6 isles.

\subsubsection{Automatic tunnel}

An automatic tunnel permits communicating an IPv6/IPv4 node with the IPv4 infrastructure without the need to configure destination tunnels, that is, the tunnel destination address is determined by the compatible IPv4 destination address containing the IPv6 packet; this address is obtained through the Border Gateway Protocol (BPG) routing protocol, which indicates the next packet hop.

\section{- $\quad$ Topology in GNS3}

For this network topology, the R1 to R4 automatic tunnel is generated and vice versa (Figure 4).

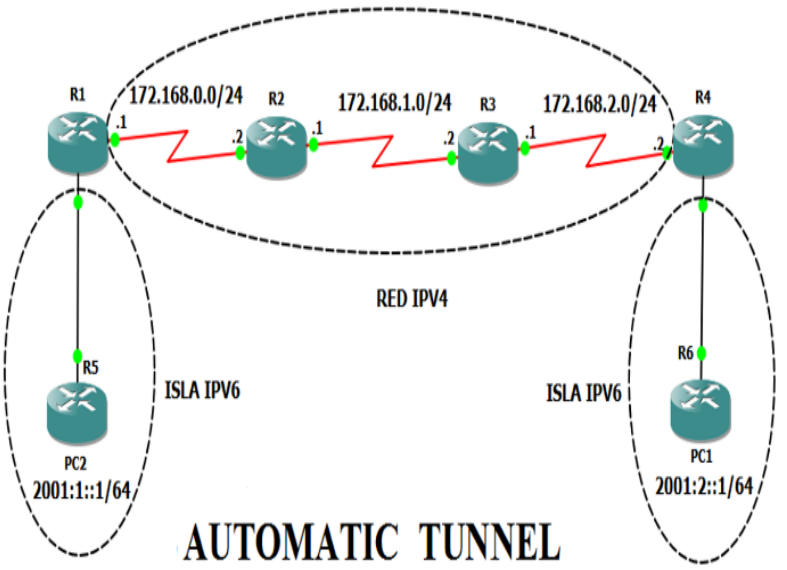

Figure 4. Automatic tunnel topology in GNS3

The R1 and R4 routers function as IPv6/IPv4 nodes, where the tunnel transporting the IPv6 packet is created by an IPv4 network. The centre routers (R2 and R3) are only IPv4 and the $\mathrm{R} 5$ and R6 routers function as IPv6 PCs. 


\section{TECCIENCIA}

- Tunnel configuration

The numerals contained by R1 are the necessary parameters to create the automatic tunnel (Figure 5). The following explains each of the items.

$>\quad$ (1) Interface tunnel0: the tunnel is created; in this case a value of 0 is assigned.

(2) IPv6 RIP 6bone enable: enables the RIP protocol RIP in IPv6.

(3) Tunnel source serial0/0: indicates the tunnel source in IPv4; for this case the serial0/0.

(4) Auto-tunnel IPv6IP tunnel mode: indicates that the IPv6 tunnel will be automatically created on $\mathrm{IPv} 4$, that is, it is not necessary to configure the tunnel's final address.

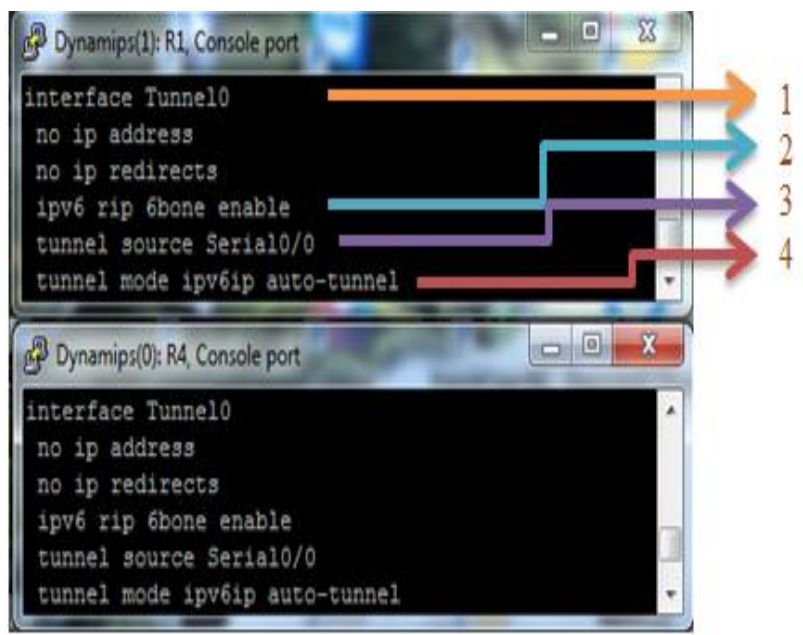

Figure 5. Automatic tunnel configuration

\subsection{RFC 30566 to 4 tunnel}

The 6 to 4 tunnel is a router to router tunnel form, which uses a (2002::/16) prefix, a prefix is assigned by IANA to specify a group of addresses to indicate they are of the same type; for this case it indicates the group of addresses from the 6 to 4 Tunneling [8].

The 6 to 4 Tunneling permits the IPv6 isles to communicate with other IPv6 isles with a minimum configuration. An IPv6 isle will be assigned a global address with a 2002:IPv4Address::/48 prefix where IPv4address is the router output interface address. This prefix has the same format as a normal prefix (::/48), which is why it permits an IPv6 domain to use it as if it were another valid prefix. In a 6 to 4 tunnel the tunnel end configuration is not necessary. The IPv4 address of each end of the tunnel is determined upon extracting the total IPv6 prefix of the destination address of the IPv6 packet that will be sent through the network [8].

\subsection{Topology in GNS3}

In the 6 to 4 tunnel topology, the tunnel is created in the $R I$ and $R 4$ routers and vice versa. The $R 2$ and $R 3$ routers are only IPv4 and the $R 5$ and $R 6$ routers are IPv6 and simulate being two PCs (Figure 6).

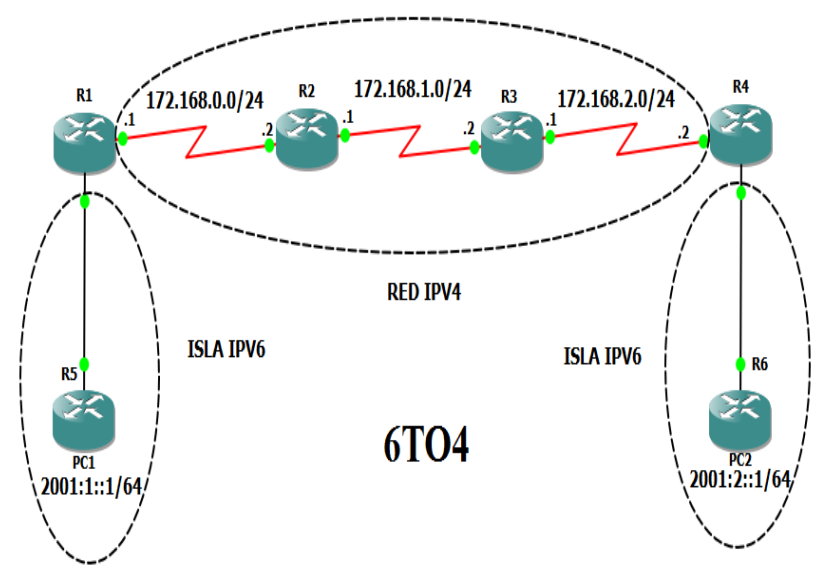

Figure 6. Topology in GNS3 of the 6 to 4 tunnel

\subsection{Tunneling configuration}

The following explains in detail the 6 to 4 tunnel configuration in the $R 1$ and $R 2$ routers, as shown in Figure 7.

$>$ (1) Interface tunnel0: the tunnel is created; in this case a value of 0 is assigned.

(2) IPv6 Address 2002:ACA8:1::/128: this IPv6 address is made up in the following manner:

The $2002(:: / 16)$ is an address assigned by IANA, then the IPv4 address from the router is added, in this case 172.168.0.1, but first the IPv4 address is converted to hexadecimal format in the following manner:

$$
\begin{array}{ll}
\text { - } & 172=A C \\
\text { - } & 168=A 8 \\
\text { - } & 00=00 \\
\text { - } & 01=01
\end{array}
$$

Then, the new address is constructed, that is, 2002:ACA8:1::/48; to finish constructing the IPv6 address an interface identifier is assigned.

- IPv6

- Address: 2002:ACA8:1::/128 


\section{TECCIENCIA}

$>$ (3) Tunnel source 172.168.0.1: indicates the tunnel source in IPv4, for this case the IPv4 address from serial0/0.

$>\quad$ (4) Tunnel mode IPv6 IP6to4: indicates the IPv6 tunnel will be created on IPv4 with the principles of the 6 to 4 tunnel.

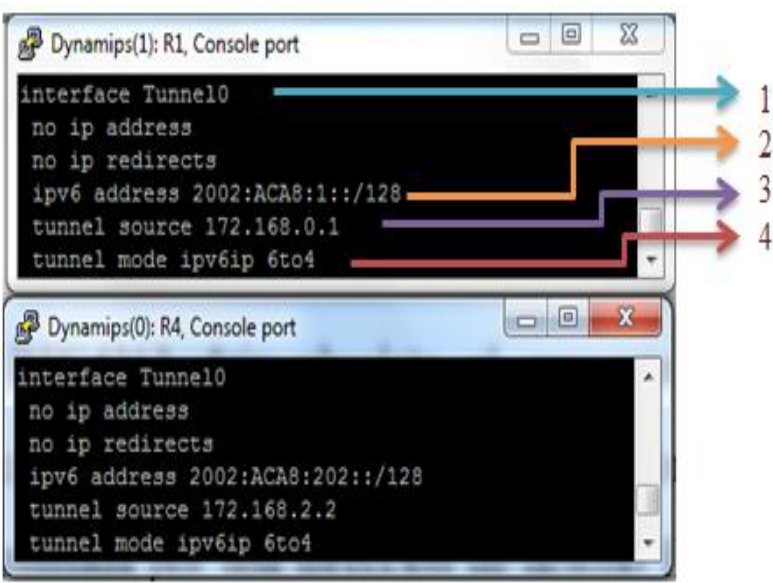

Figure 7. Tunnel configuration

\subsubsection{Address translation}

The RFCs 2765 and 2766 describe the address and protocol translation. The objective of this transition mechanism consists in providing transparent routes to the nodes in the networks or IPv6 isles to communicate them the nodes of IPv4 networks and vice versa.

- Translation of the IPv6 header to IPv4: When an IPv6 packet reaches an IPv6/IPv4 node it translates the address and the protocol and turns it into an IPv4 packet.

2.6.2. Network Address Translation - Protocol Translation (Nat-Pt)

Nat-Pt, according to RFC 2766, is a Stateless IP/ICMP Translation (SIIT) application, which provides a transparent route between IPv6 and IPv4 networks. That is, it appears as a link gate between two networks; additionally, it is in charge of translating all the addresses and protocols of the packets passing through it [9].

This technique requires at least an IPv4 available address for the IPv6 network seeking communication with IPv4 networks. It also uses a mapping table that contains the relationship of the IPv6 addresses and prefixes with the IPv4 addresses to provide a transparent route.

\subsection{Topology in GNS3}

For the Nat-Pt topology in GNS3, the IPv6 PCs are R5 and R6 routers; the R2 and R3 routers are only IPv4; and R1 and R4 routers are IPv6/IPv4 nodes.

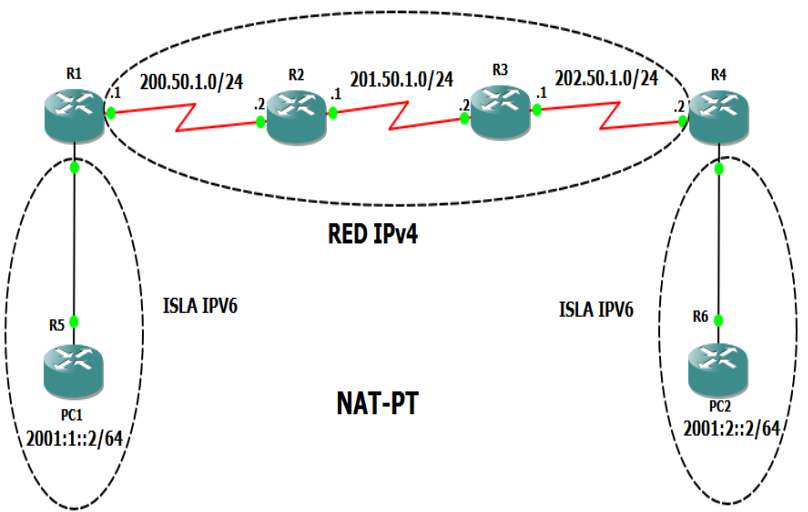

Figure 8. 6 to 4 tunnel topology in GNS3

\subsubsection{Mapping table configuration}

Figure 9 describes how the IPv6 and IPv4 origin and destination addresses are translated; for example, in R1 it is described, thus:

$>$ IPv4 origin address: 200.50 .1 .3

$>$ IPv6 origin address: 2001:1::2

$>$ IPv4 destination address: 202.50.1.3

$>$ IPv6 destination address: 2010::ABCD

That is, when a packet reaches R1 with the 2010::ABCD address, it changes address and protocol for an IPv4 address 202.50.1.3 so it can be transported by the IPv4 network, and when a packet reaches R1 with address 200.50.1.3, it translates it into the IPv6 address 2001:1::2, that is, it sends it to PC1. This same procedure is carried out in R4 for the protocol and address translation and, thus IPv6 packets can travel through an IPv4 network.

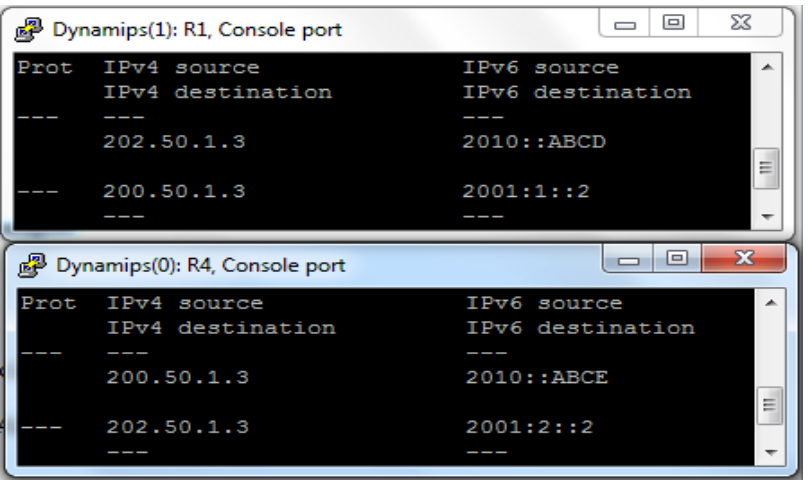

Figure 9. Mapping table configuration 


\section{TECCIENCIA}

\subsection{Automatic Tunneling tests in GNS3}

\section{Results}

\section{1 experimental design}

A set of tests were designed to analyze the performance of the simulations implemented. By sending 200 packets of different length, we evaluated the network's performance upon transporting packets. We also examined the behavior of the IPv6/IPv4 node memory, given that it is in charge of making the IPv6 and IPv4 protocols compatible and can use the IPv4 network.

For the memory tests, it must be remarked that free memory of the tunneling-type techniques, that is, the manual, automatic, and 6 to 4 tunnels was $69,376,000$ Bytes; for the address translation technique the memory was 46,128,512 Bytes. Stemming from these data, a relationship is made between memory used and free memory; likewise, a relationship is made in the percentage of CPU consumption used by the router to send data.

The data shown ahead were obtained for the ROUND$T R I P(m s)$ in R5 and for R1 memory use; the first indicates the time spent by the packets to reach their destination and the latter is in charge of encapsulating or translating an IPv6 packet into IPv4.

\subsection{Manual Tunneling tests in GNS3}

Figure 10 shows the manual tunneling simulation latency behavior.

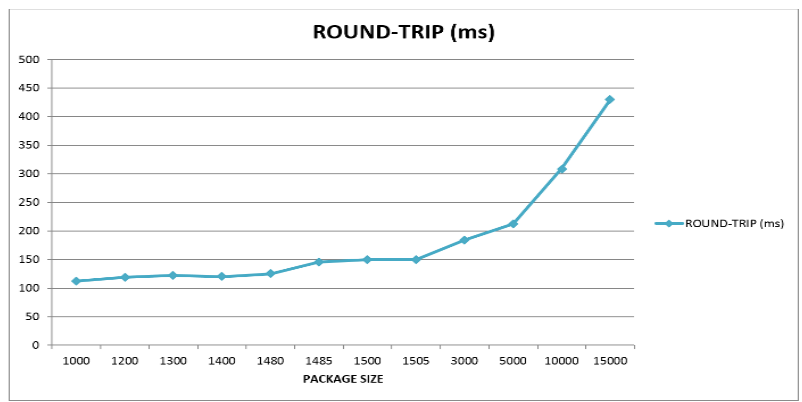

Figure 10. Latency of manual tunneling

Figure 11 details the router's (R1) memory use.

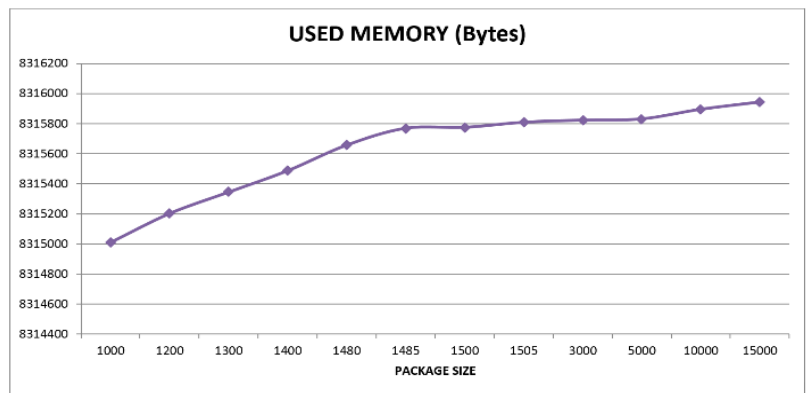

Figure 11. R1 memory use
Figure 12 shows the latency (Round-Trip) of the automatic tunneling.

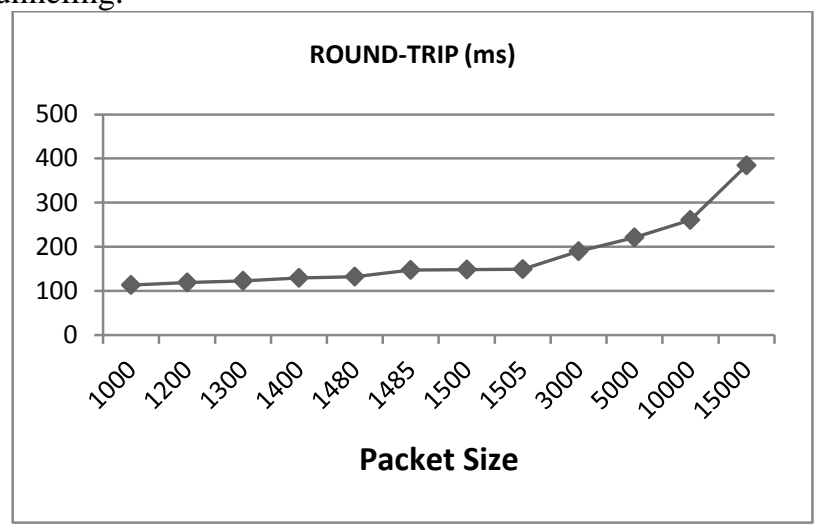

Figure 12. Latency of automatic Tunneling

Figure 13 shows the router's (R1) memory use, according to packet size.

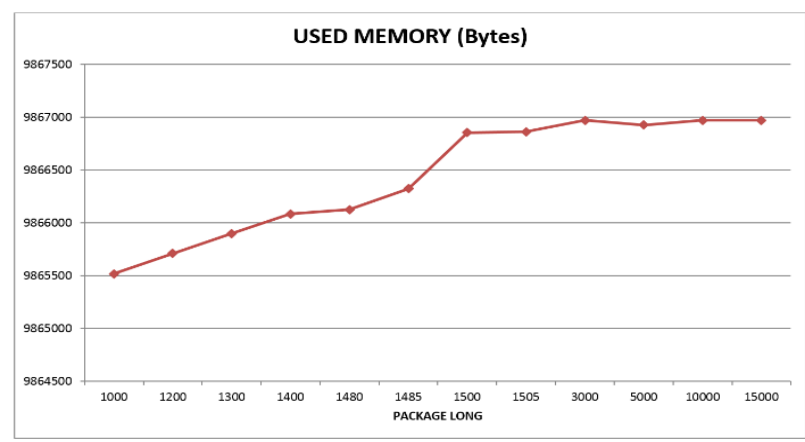

Figure 13. R1 memory use

\subsection{Tests of 6 to 4 Tunneling in GNS3}

The following shows the 6 to 4 tunneling simulation latency behavior (Figure 14).

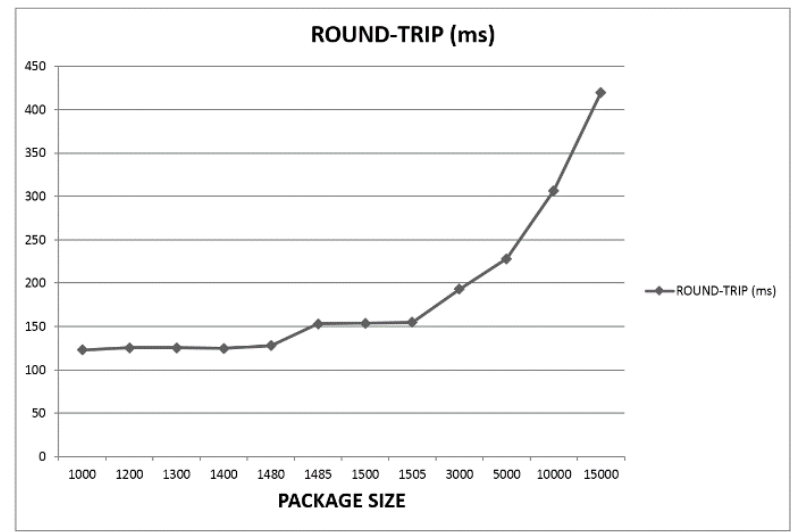

Figure 14. 6 to 4 tunneling latency behavior 


\section{TECCIENCIA}

Figure 15 shows the router's (R1) memory use, according to packet size.

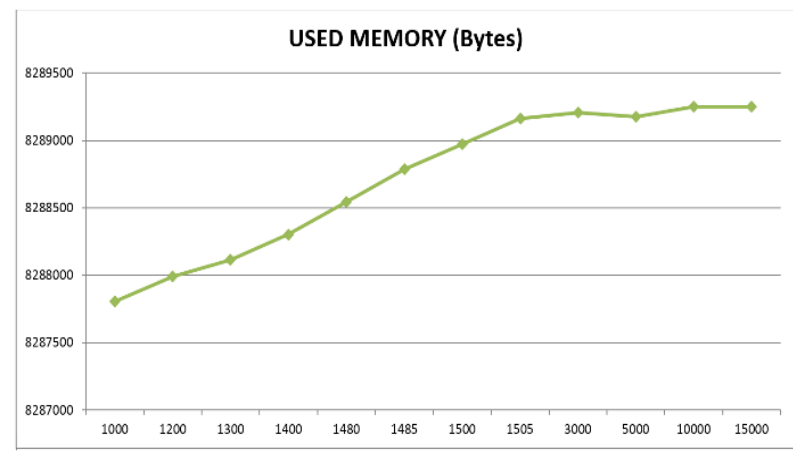

Figure 15. R1 memory use

\subsection{Nat-Pt tests in GNS3}

Figure 16 shows the Nat-Pt simulation latency behavior.

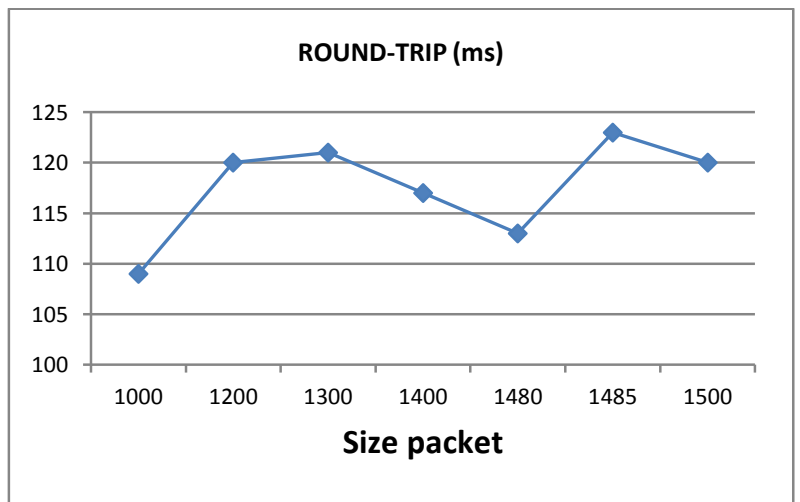

Figure 16. Nat-Pt latency

The following shows the R1 memory behavior when it translates the IPv6 packets in IPv4.

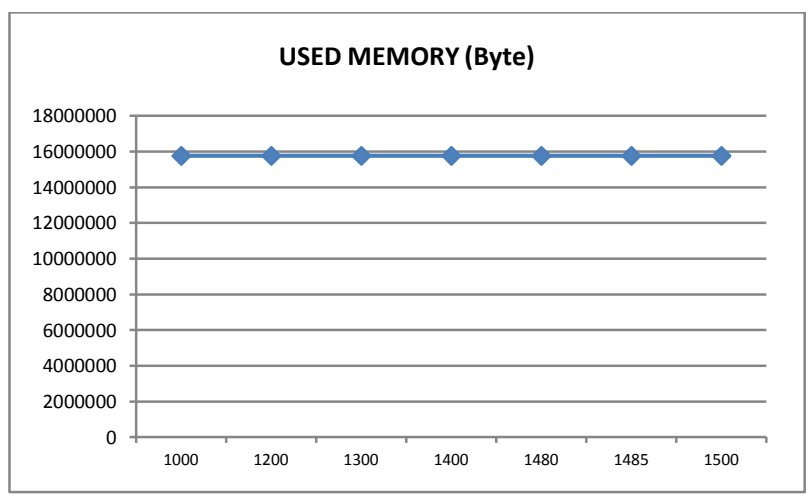

Figure 17. R1 memory use

\subsection{Wireshark tests}

Table 1. Show the communication between PC1 and PC2. First, a petition request is sent from $\mathrm{PC} 1$ to $\mathrm{PC} 2$; then, $\mathrm{PC} 2$ sends a response to $\mathrm{PC} 1$. That is, communication and data delivery can occur between them.

Table 1. Communication between PC1 and PC2

\begin{tabular}{|c|c|c|}
\hline Source & Destination & Protocol \\
\hline $2001: 1: 1$ & $2001: 2: 1$ & ICMPV6 \\
\hline $2001: 2: 1$ & $2001: 1: 1$ & ICMPV6 \\
\hline $2001: 1: 1$ & $2001: 2: 1$ & ICMPV6 \\
\hline $2001: 2: 1$ & $2001: 1: 1$ & ICMPV6 \\
\hline $2001: 1: 1$ & $2001: 2: 1$ & ICMPV6 \\
\hline $2001: 2: 1$ & $2001: 1: 1$ & ICMPV6 \\
\hline $2001: 1: 1$ & $2001: 2: 1$ & ICMPV6 \\
\hline $2001: 2: 1$ & $2001: 1: 1$ & ICMPV6 \\
\hline
\end{tabular}

Tunneling is based on encapsulating IPv6 packets in an IPv4 header to be sent through an IPv4 network; Figure 18 details the IPv4 header.

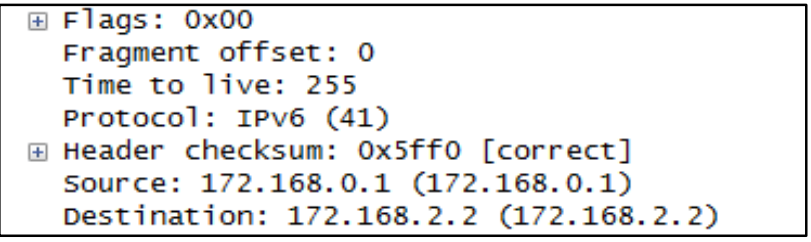

Figure 18. IPv4 header in Wireshark

The previous figure shows the IPv4 header, highlighting the following information:

$>$ The address of origin is the interface of the encapsulation node, which is address 172.168.0.1

$>$ The destination address is the interface of the deencapsulation node; for this case it is IP address 172.168.2.2

$>$ The protocol has a value of 41 , meaning that it has IPv6 information

For the IPv6 datagram, the following information is shown:

Pay1oad 1ength: 60
Next header: ICMPv6 (0x3a)
Hop 1imit: 63
Source: $2001: 1:: 1 \quad(2001: 1:: 1)$
Destination: 2001:2::1 (2001:2::1)

Figure 19. IPv6 datagram in Wireshark

The following information is highlighted from Figure 19:

$>$ The address of origin is $2001: 1:: 1$

$>$ The destination address is $2001: 2:: 1$

$>$ No registry exists of the packet having been fragmented 
In case a packet is fragmented in the automatic, manual, or 6

to 4 tunnels, it will be noted thus in Wireshark. In this case, the packets will be fragmented as of 1480 bytes, given that 20 bytes are necessary for the IPv6 header, that is, the MTU in the IPv6 tunnels on IPv4 is 1480 bytes.

Table 2. Fragmentation of IPv6 packet

\begin{tabular}{|c|c|c|c|}
\hline Source & Destination & Protocol & Length Info \\
\hline $2001: 1:: 1$ & $2001: 2:: 1$ & IPv6 & $\begin{array}{c}\text { 1504 IPv6 fragment } \\
\text { (nxt=ICMv6 (0x3a) off=0 } \\
\text { id=0xa) }\end{array}$ \\
\hline $2001: 1:: 1$ & $2001: 2:: 1$ & ICMPv6 & $\begin{array}{c}\text { 100 Echo (Ping) request } \\
\text { id=0x1979, seq=0 }\end{array}$ \\
\hline $2001: 2:: 1$ & $2001: 1:: 1$ & IPv6 & $\begin{array}{c}\text { 1504 IPv6 fragment } \\
\text { (nxt=ICMv6 (0x3a) off=0 } \\
\text { id=0x9) }\end{array}$ \\
\hline $2001: 2:: 1$ & $2001: 1:: 1$ & ICMPv6 & $\begin{array}{c}\text { 100 Echo (Ping) request } \\
\text { id=0x1979, seq=0 }\end{array}$ \\
\hline $2001: 1:: 1$ & $2001: 2:: 1$ & ICMPv6 & $\begin{array}{c}\text { 1504 IPv6 fragment } \\
\text { (nxt=ICMv6 (0x3a) off }=0 \\
\text { id=0xb) }\end{array}$ \\
\hline $2001: 2:: 1$ & $2001: 1:: 1$ & IPv6 61 (Ping) request & $\begin{array}{c}\text { 1504 IPv6 fragment } \\
\text { (nxt=ICMv6 (0x3a) off=0 } \\
\text { id=0xa) }\end{array}$ \\
\hline $2001: 2:: 1$ & $2001: 1:: 1$ & ICMPv6 & $\begin{array}{c}100 \text { Echo (Ping) request } \\
\text { id=0x1979, seq=0 }\end{array}$ \\
\hline
\end{tabular}

The previous figure shows that the IPv6 packet has been fragmented and the packets have been delivered from PC1 to PC2. When the IPv6 packet is fragmented, the datagram fields remain in the following manner (Figure 22).

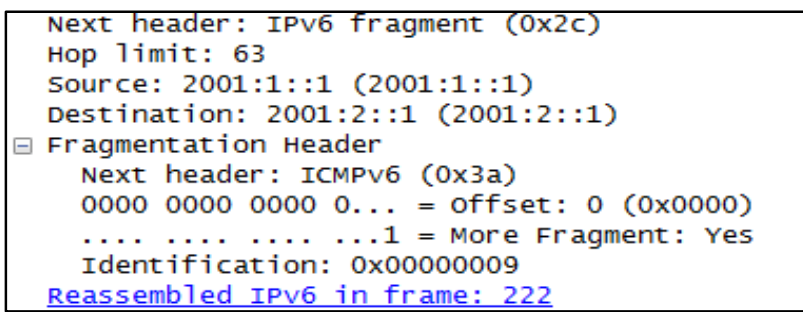

Figure 20. Fragmented IPv6 datagram

The following information is highlighted:

a) The Next Header field indicates the IPv6 packet has been fragmented.

b) The Header fragmentation field indicates that the option with the most fragments is in 1 , that is, the packet is fragmented.

c) The destination and origin addresses do not change.

In the NAT-PT technique the address and the IPv6 protocol are translated into $\mathrm{IPv} 4$, that is, the packet will travel with an IP address assigned in the mapping that contains the IPv6/IPv4 router (Table 3).

Table 3 PC1 and PC2 Communication

\begin{tabular}{|c|c|c|c|c|}
\hline Source & Destination & Protocol & Length & Info \\
\hline 200.50 .1 .3 & 202.50 .1 .3 & ICMP & $\begin{array}{c}84 \text { Echo } \\
\text { (ping) }\end{array}$ & Request \\
\hline 200.50 .1 .3 & 202.50 .1 .3 & ICMP & $\begin{array}{c}84 \text { Echo } \\
\text { (ping) }\end{array}$ & Reply \\
\hline 200.50 .1 .3 & 202.50 .1 .3 & ICMP & $\begin{array}{c}84 \text { Echo } \\
\text { (ping) }\end{array}$ & Request \\
\hline 200.50 .1 .3 & 202.50 .1 .3 & ICMP & $\begin{array}{c}84 \text { Echo } \\
\text { (ping) }\end{array}$ & Reply \\
\hline 200.50 .1 .3 & 202.50 .1 .3 & ICMP & $\begin{array}{c}84 \text { Echo } \\
\text { (ping) }\end{array}$ & Request \\
\hline
\end{tabular}

Figure 21 illustrates the IPv4 header assigned in the router, in this case $\mathrm{R} 1$, the most relevant information is:

$>$ The address of origin is 200.50.1.3, which is an available address in the $\mathrm{R} 1$ router network.

$>$ The destination address is 202.50.1.3, which is an available address in the $\mathrm{R} 4$ router network.

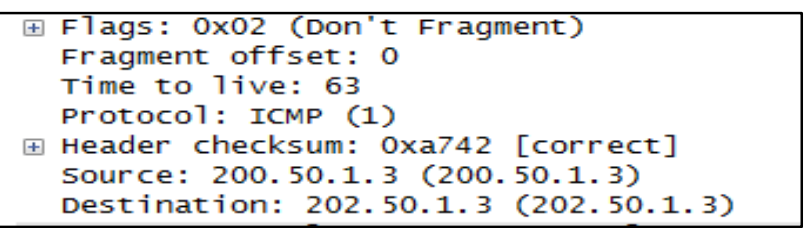

Figure 21. IPv4 datagram in Wireshark

When packet sent from $\mathrm{PC} 1$ to $\mathrm{PC} 2$ reaches the final router in this case R4 translates the IPv4 packet coming through the network into a IPv6 packet to be delivered to its destination.

\section{Discussion}

The GNS3 Emulator is a useful tool for the business environment because it reduces implementation costs, given that in GNS3 it is possible to experiment and test the different functionalities the Cisco IOS contain to avoid causing any damage in real devices. This emulator uses hardware resources no other simulator like Packet Tracer uses; both its graphic interface and its results assimilate real-life devices.

An aspect referring to the simulation was the application of the router to router transition mechanisms to the different topologies, given that the internet service providers (ISPs) are in charge of covering and providing adequate service to its users, depending on their needs because users are indifferent to the protocols and means used by their network to carry out tasks like making payments, sending e-mails, or browsing different web pages. Due to this, we discarded the de host to host and host to router applications.

\section{Conclusions}




\section{TECCIENCIA}

The simulations carried out comply perfectly with the functions required to transmit IPv6 data through IPv4 networks.

The transition mechanisms were designed for the interaction of the two IPv6 and IPv4 protocols, while managing the total deployment or emigration to the IPv6 network that can take several years.

The main reason to migrate to IPv6 is due to the depletion of IP addresses, this new protocol improves certain IPv4 parameters, like higher efficiency to send data and support to communication security.

The advantages offered by manual Tunneling and automatic Tunneling are that not many resources are consumed, and it is a transparent method at IPv6 level with which applications are not affected.

The setbacks of manual Tunneling are the manual configuration and not in dynamic manner of the final nodes of Tunneling, given that to share information with other networks; the nodes must be previously configured for said networks.

The advantages offered by the 6 to 4 Tunneling is that Tunneling is only established when necessary, Tunneling are dynamic and it is not necessary to configure them, each IPv6 network only needs an IPv4 global address; we can have up to $2^{32}$ IPv6 networks.

The inconvenience presented by the 6 to 4 Tunneling is that to create a Tunneling only the $2002:: / 16$ prefix can be used.

The NAT-PT technique has fragmentation problems, which is a disadvantage when sending large packets, given that it needs the support of other techniques to carry out a correct fragmentation. Likewise, the translation process is more costly in the use of resources than the application of tunnels.

For WAN networks, the maximum latency that can be worked with without problems is $380 \mathrm{~ms}$, after this time packets start getting lost. In the Tunneling technique when the packet was too large (15000 bytes) losses started to be produced and the packets were not totally delivered and their times exceeded $380 \mathrm{~ms}$.

It is more effective to use dynamic tunnels (Automatic tunnel and 6to4) to interconnect IPv6 isles through IPv4 than the manual Tunneling techniques, given that with a higher number of isles less final nodes need to be configured.
The GNS3 program is an easy to install tool, it is open software and permits developing work in the business sector, given that it reduces the cost implementing the networks; it is also useful in the student sector because it is quite accessible to the study of networks, it also works jointly with Wireshark for packet capture.

To implement any transition mechanism, bear in mind the company's or user's infrastructure, and what would be the application because some equipment does not support the IPv6 protocol or they do not have sufficient resources for its implementation.

\section{References}

[1] G. Huston, «Transitioning Protocols - Part 1,» February 2011. [En línea]. Available: http://www.potaroo.net/ispcol/201102/transtools-part1.pdf.

[2] M. Qaissaunee, «Frequently Asked Q,» 04 January 2011. [En línea]. Available: http://q-ontech.blogspot.com/2011/01/story-ofipv5.html. [Último acceso: January 2012].

[3] T. Wiegand, G. Sulliva

n, G. Bjontegaard y A. Luthra, «Overview of the H.264/AVC Video Coding Standard,»IEEE TRANSACTIONS ON CIRCUITS AND SYSTEMS FOR VIDEO TECHNOLOGY, vol. 13, $\mathrm{n}^{\circ}$ 7, pp. 560-576, 2003

[4] A. E. Salinas, Interviewee, Protocolo IPv6: Presente y futuro de la internet. [Entrevista]. 23 March 2011.

[5] IETF, «Request fot comments (RFC),» IETF, [En línea]. Available: http://www.ietf.org/rfc.html. [Último acceso: MAY 2011].

[6] IETF, «Basic Transition Mechanisms for IPv6 Hosts and Routers,» Sun Microsystems, October 2005. [En línea]. Available: https://tools.ietf.org/html/rfc4213.

[7] I. Minei y J. Lucek, MPLS-Enabled Applications: Emerging Developments and New Technologies, Wiley Editorial, 2005.

[8] J. Rosenber, H. Schulzrinne, G. Camarillo, A. Johnston, J. Peterson, R. Sparks, M. Handley y E. Schooler, « SIP: Session Initiation Protocol,» June 2002. [En línea]. Available: https://www.ietf.org/rfc/rfc3261.txt.

[9] ETSI- Telecoms \& Internet converged Services \& Protocols for Advanced Network, «TISPAN- Defining the next generation network,» European Telecommunications Standards Institute, [En línea]. Available: http://www.etsi.org/tispan/.

[10] ITU-T, «Packet- Based Multimedia Communications Systems,» ITU-T Recomendation H.323, January 1998. [En línea]. Available: http://www.itu.int/rec/T-REC-H.323/es. 